\title{
Two-colour QCD at finite density with two flavours of staggered quarks
}

\author{
Lukas Holicki*i \\ Institut für Theoretische Physik, Justus-Liebig-Universität Gießen, 35392 Gießen, Germany \\ E-mail: Lukas.Holicki@physik.uni-giessen.de \\ Jonas Wilhelm \\ Institut für Theoretische Physik, Justus-Liebig-Universität Gießen, 35392 Gießen, Germany \\ E-mail: Jonas.Wilhelmephysik.uni-giessen.de
}

Dominik Smith

Institut für Theoretische Physik, Justus-Liebig-Universität Gießen, 35392 Gießen, Germany

E-mail: Dominik.D.Smith@theo.physik.uni-giessen.de

\section{Björn Wellegehausen}

Institut für Theoretische Physik, Justus-Liebig-Universität Gießen, 35392 Gießen, Germany

E-mail: Bjoern.Wellegehausen@theo.physik.uni-giessen.de

\section{Lorenz von Smekal}

Institut für Theoretische Physik, Justus-Liebig-Universität Gießen, 35392 Gießen, Germany

E-mail: Lorenz. Smekal@theo.physik.uni-giessen.de

In this contribution we revisit simulations of two-color QCD with rooted staggered quarks at finite density, where baryon-number spontaneously breaks and a diquark condensate forms. We thereby pay special attention to simulating outside the lattice-artifact bulk phase, in which $Z_{2}$ monopoles condense, and investigate some of the consequences of this, e.g. on the chiral and the diquark condensate which were known to be well described by chiral effective field theory. Not surprisingly, on finer lattices outside the bulk phase the quark condensate now requires additive renormalization before it can be compared with effective field theory predictions. The subtraction must necessarily depend on the chemical potential, however. The diquark condensate is not affected by this problem and remains in good agreement with these predictions. We also compare staggered with Wilson quarks to demonstrate that the two fermion discretizations yield qualitatively different results well below half-filling already. We close with prelimiary results for the Goldstone spectrum to demonstrate that the continuum pattern is recovered also with staggered quarks outside the bulk phase.

34th annual International Symposium on Lattice Field Theory

24-30 July 2016

University of Southampton, UK

\footnotetext{
* Speaker.

${ }^{\dagger}$ This work was supported by the Helmholtz International Center for FAIR within the LOEWE initiative of the State of Hesse.
} 


\section{Introduction}

Lattice simulations of two-colour $\mathrm{QCD}\left(\mathrm{QC}_{2} \mathrm{D}\right)$ can be performed at finite density without a sign problem and by now have a long history already $[1,2]$. The physics of the bosonic diquark baryons is believed to be fairly well understood and qualitatively resembles QCD at finite isospin density with pion condensation [3]. There is good guidance from effective field theory and random matrix theory predictions $[4,5,6]$ and model studies of the BEC-BCS crossover inside the condensed phase [7, 8]. $\mathrm{In}_{\mathrm{QC}_{2}} \mathrm{D}$ diquarks play a dual role as two-color baryons and pseudo-Goldstone bosons of the dynamical breaking of an extended chiral symmetry. When they condense they are expected to form a superfluid which changes in nature from a Bose-Einstein condensate of tightly bound diquarks to a BCS-like pairing of quarks as chiral symmetry gets gradually restored with increasing density. Recent results with both, staggered [9] and Wilson [10] quarks have confirmed previous evidence from coarser lattices for this superfluid phase. In line with the predictions from chiral effective Lagrangians, at zero temperature it first occurs when the quark chemical potential $\mu$ reaches half the pion mass, and this is also correctly reproduced in effective lattice theories for heavy quarks [11], from the same combined strong-coupling and hopping expansion techniques that are actively being developed for cold and dense QCD [12].

Here, we revisit the low-temperature part of the $\mathrm{QC}_{2} \mathrm{D}$ phase diagram with rooted staggered quarks. After reproducing previous results for the chiral- and diquark-condensates, the quarknumber density and the Goldstone spectrum along the $\mu$ direction at $\beta=1.5$, we assess the influence of the lattice-artifact bulk phase dominated by $Z_{2}$ monopoles. With an improved gauge action and a somewhat larger lattice coupling of $\beta=1.7$ on the other hand, these monopoles are sufficiently suppressed to connect with continuum results. While this then requires a $\mu$-dependent additive renormalization of the chiral condensate, the extrapolated diquark condensate and quarknumber density near the onset transition at half the pion mass remain in very good agreement with the effective field theory predictions. Beyond that one might speculate to see evidence of the BECBCS crossover at larger $\mu$, but this gets hard to disentangle from discretization artifacts at larger $\mu$ which we assess by comparing staggered with Wilson fermions revealing significant qualitative differences between the two fermion discretizations already well below half-filling.

Finally but maybe most importantly our prelimiary Goldstone spectrum near the onset transition provides good evidence that the symmetry breaking pattern of $\mathrm{QC}_{2} \mathrm{D}$ in the continuum is recovered also with staggered quarks outside the bulk phase and hence in the continuum limit, despite the wrong antiunitary symmetries of staggered fermions at finite lattice spacing $[4,5]$.

\section{General Setup}

To study the spontaneous breaking of baryon number on a finite lattice, one adds the diquark source term $\sim \lambda$ to the Lagrangian as an explicitly symmetry-breaking external field. The staggered fermion action of $\mathrm{QC}_{2} \mathrm{D}$ is then expressed in a Nambu-Gorkov basis as [2]

$$
S_{f}=\bar{\chi} D(\mu) \chi+\frac{\lambda}{2}\left(\chi^{T} \tau_{2} \chi+\bar{\chi} \tau_{2} \bar{\chi}^{T}\right)=\frac{1}{2}\left(\bar{\chi}, \chi^{T} \tau_{2}\right) \underbrace{\left(\begin{array}{cc}
\lambda & D(\mu) \\
-D^{\dagger}(\mu) & \lambda
\end{array}\right)}_{=: A}\left(\begin{array}{c}
\tau_{2} \bar{\chi}^{T} \\
\chi
\end{array}\right),
$$


where $D$ denotes the staggered Dirac operator, such that $\operatorname{det} A=\operatorname{det}\left(D^{\dagger}(\mu) D(\mu)+\lambda^{2}\right)$. Results are extrapolated to $\lambda \rightarrow 0$, and the diquark condensate is obtained from

$$
\langle q q\rangle=\left.\left\langle\chi^{T} \tau_{2} \chi\right\rangle \propto \frac{\partial \ln Z}{\partial \lambda}\right|_{\lambda \rightarrow 0} .
$$

We use the RHMC algorithm [13] to simulate a rooted fermion $\operatorname{determinant} \operatorname{det} A^{N_{f} / 8}$.

The prediction from the leading order chiral Lagrangian with diquark source $\lambda$ for fundamental staggered quarks in $\mathrm{QC}_{2} \mathrm{D}$ is that the vaccum rotates at fixed $\langle\bar{q} q\rangle^{2}+\langle q q\rangle^{2}$ from a chiral to a diquark condensate with $\tan \phi=\lambda / m$ and rotation angle $\alpha(\mu)$ as $\mu$ increases, from $\alpha=\phi$ for $\mu=0$ to $\alpha=\pi / 2$ for $\mu \gg \mu_{c}=m_{\pi} / 2$, which is obtained from [4]

$$
\begin{array}{ccc}
4 \mu^{2} \cos \alpha \sin \alpha=m_{\pi}^{2} \sin (\alpha-\phi), & \text { such that } & n_{B}=8 N_{f} F^{2} \mu \sin ^{2} \alpha, \\
\langle\bar{q} q\rangle=2 N_{f} G \cos \alpha, & \text { and } \quad\langle q q\rangle=2 N_{f} G \sin \alpha .
\end{array}
$$

We fit these equations to the results from our simulations. For $\lambda \rightarrow 0$ they describe the zerotemperature onset of diquark condensation at $\mu_{c}=m_{\pi} / 2$, which is determined as a fit parameter here and compared with an independent determination of $m_{\pi}$ from spectroscopy as a check.

\section{Results in the bulk phase}

After reproducing the results from [5] for $\langle q q\rangle,\langle\bar{q} q\rangle$ and the quark number-density $\langle n\rangle$ with $N_{f}=4$ at $\beta=1.5, N_{s}=12, N_{t}=24, a m=0.025, a \lambda=0.0025$ as a check, we have performed the analogous simulations with the fourth root for $N_{f}=2$, see Fig. 1. The corresponding Goldstone spectrum is shown in Fig. 2. As for the $N_{f}=4$ results from [5] it reflects the symmetry breaking pattern of fundamental staggered quarks (shown here as $\chi$ PT fit) which resembles that of adjoint QCD or $G_{2}$-QCD in the continuum as most notably seen in the behavior of the pion branch above the onset that here results at $a \mu_{c}=0.18889(45)$ from the fit. Within the errors this well agrees with our spectroscopic result from the pion correlator at $\mu=0$ which yields $a m_{\pi} / 2=0.1887(06)$.

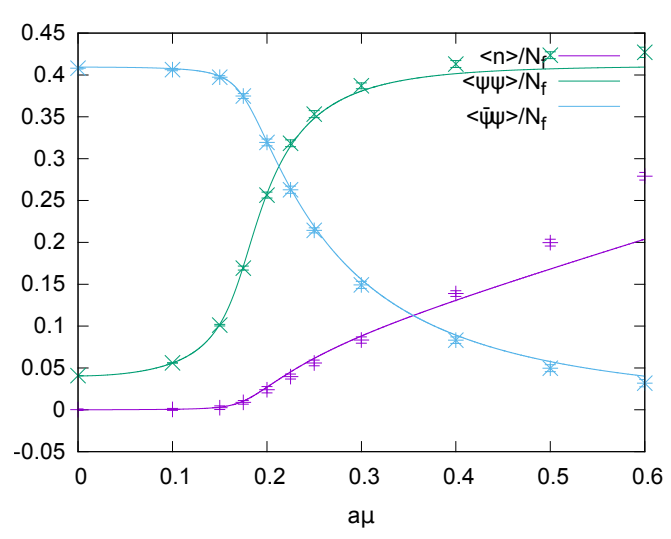

Figure 1: Fit of lattice data to $\chi \mathrm{PT}$ predictions of condensates and density at $\beta=1.5$.

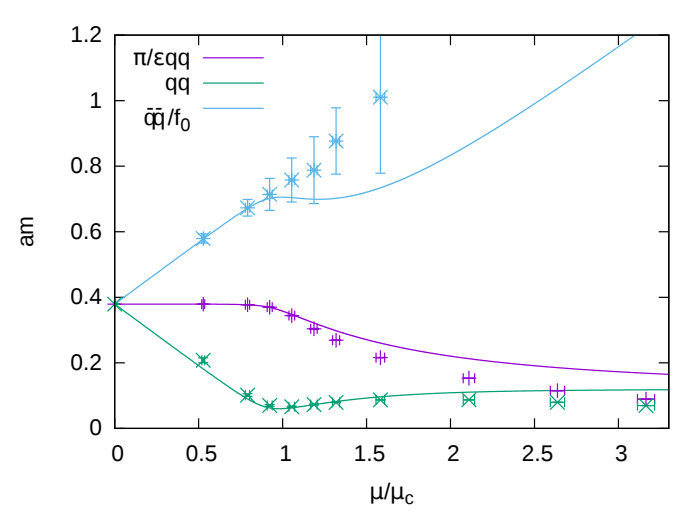

Figure 2: Goldstone spectrum for Fig. 1 without disconnected contributions in $\bar{q} \bar{q} / f_{0}$.

The scalar $f_{0}$ mixes with the heavy (anti)diquark mode when baryon number is broken in the diquark-condensation phase. The discrepancy of the corresponding $\bar{q} \bar{q} / f_{0}$ branch with $\chi \mathrm{PT}$ there is to a large extend due to disconnected contributions to $f_{0}$ which we did not calculate here. 
A potential problem in these simulations is the lattice-artifact bulk phase of $\mathrm{SU}(2)$ with $Z_{2^{-}}$ monopole condensation at strong coupling [14]. We have therefore measured the $Z_{2}$-monopole density $z=1-\frac{1}{N_{c}} \sum_{c} \prod_{P \in \partial c} \operatorname{sgn}(\operatorname{tr} P)$ sensitive to preferred signs of plaquettes on the faces of elementary cubes $c$. Its dependence on the lattice coupling with and without quarks is shown in Fig. 3. It is rather insensitive to unquenching, at $\beta=1.5$ even with $a m=0.01$ (and $\mu=0$ ) we obtain $\langle z\rangle=0.8840$ (1) which shows that this is well below the crossover and deep inside the bulk phase.

To suppress $Z_{2}$ monopoles we therefore use a tree-level Symanzik-improved gauge action [15], cf. Fig. 3. From extended meson spectroscopy [16] we conclude that the best compromise between discretization and finite-volume effects with the improved action on our present $16^{3} \times 32$ lattice is obtained for $\beta=1.7$, see Fig. 4. The $Z_{2}$-monopole density is then down to $\langle z\rangle=0.2734$ (7).

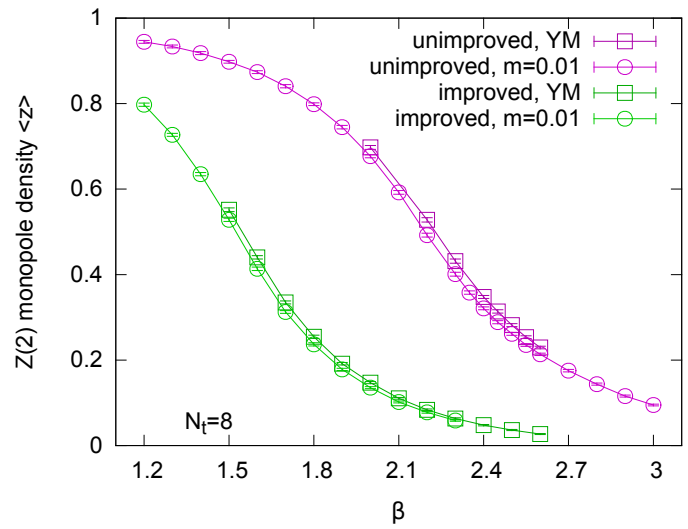

Figure 3: $Z_{2}$-monopole density, $N_{s}=12, N_{t}=8$.

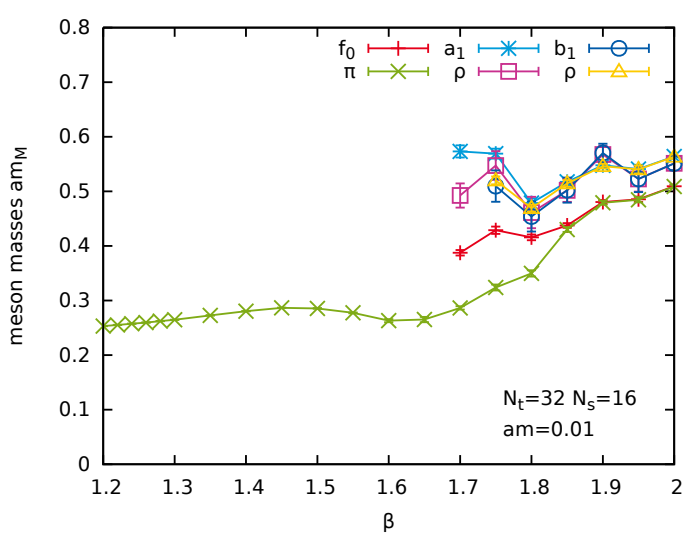

Figure 4: Meson masses over $\beta$ from [16].

\section{Chiral and diquark condensates outside the bulk phase}

With the improved gauge action, $\beta=1.7$ and $a m=0.01$ on the $16^{3} \times 32$ lattice, where $m_{\pi} / m_{\rho}=$ $0.5816(27)$, we have then performed a low temperature scan of the phase diagram. The chiral condensate as a function of the quark chemical potential in units of $\mu_{c}$ is shown in Fig. 5a. Not surprisingly, on the finer lattice it now requires additive renormalization. In finite temperature simulations at $\mu=0$ this can be achieved with a connected susceptibility subtraction, $\Sigma=\langle\bar{q} q\rangle_{m_{q}}-m_{q} \chi^{\text {con }}$, by observing that (up to a factor $m_{q}$ ) chiral condensate and susceptibility share the same quadratic divergence $c_{\mathrm{UV}} / a^{2}$ and that this mainly comes from the connected part $\chi^{\text {con }}$ of the latter [17]. To see whether this subtraction can be used at finite $\mu$ as well, we also included our results for $\chi^{\text {con }}$ in Fig. 5a. We observe that they both drop to zero above $\mu_{c}$, so the quadratic divergence must depend on $\mu$ in the diquark condensation phase, ${ }^{1}$ i.e. $c_{\mathrm{UV}}=c_{\mathrm{UV}}(\mu)$. Secondly, and more importantly, however, we also observe evidence of a singular contribution in the connected susceptibility $\chi^{\text {con }}$ near the diquark-condensation transition at $\mu=\mu_{c}$, as seen from its volume dependence in Fig. 5b. This is different from the chiral transition where the thermodynamic singularity resides in the disconnected part of the susceptibility. It means that this singularity in $\chi^{\text {con }}$ will dominate over $c_{\mathrm{UV}} / a^{2}$ at finite $a$ in the infinite volume limit. The chiral condensate on the other hand, at zero temperature, must remain independent of $\mu$ for $\mu<\mu_{c}$. It does not have such a singular contribution

\footnotetext{
${ }^{1}$ As it also does for free lattice fermions of mass $m$ at $\mu>m$.
} 
and it would be unphysical to introduce one with the connected susceptibility subtraction. At any rate, this would introduce a $\mu$-dependence below $\mu_{c}$ and hence a Silver-Blaze problem. Therefore, a different ( $\mu$-dependent) subtraction of the chiral condensate is required at finite density.

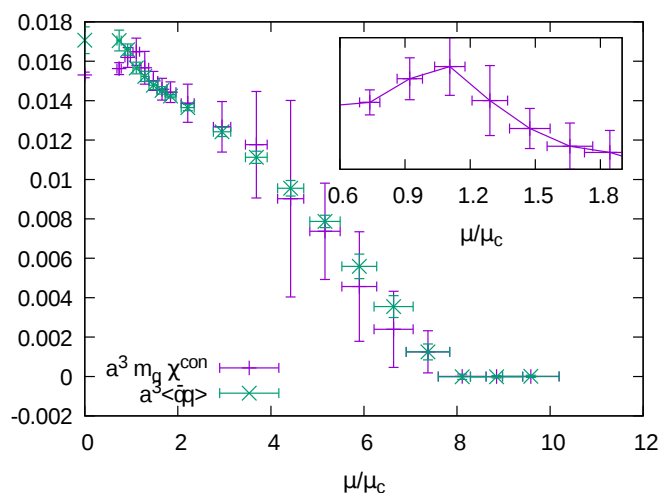

(a) $\langle\bar{q} q\rangle$ and $\chi^{\text {con }}$ with a zoom to its singular part.

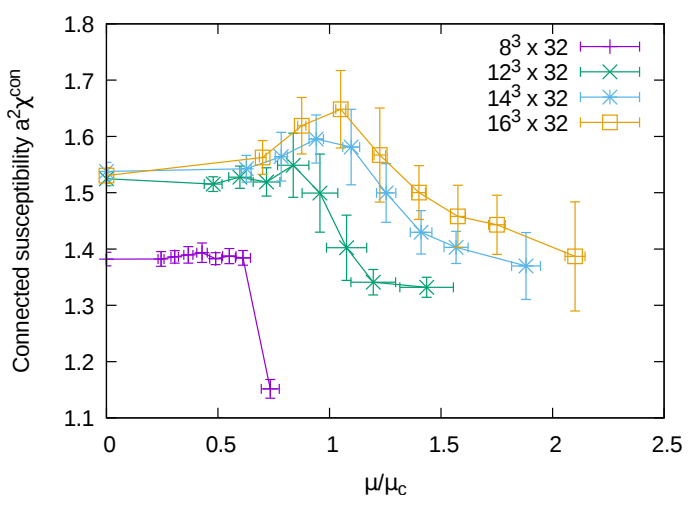

(b) volume dependence of $\chi^{\text {con }}$.

Figure 5: $\langle\bar{q} q\rangle$ and its connected susceptibility $\left(16^{3} \times 32\right.$ lattice, $\left.a m=0.01, \beta=1.7, a \lambda=0.005\right)$.

The diquark condensate in Fig. 6a is unaffected by this problem. Its $\lambda \rightarrow 0$ extrapolation now yields the diquark-condensation transition at $a \mu_{c}=0.1356(86)$ from the fit to the $\chi \mathrm{PT}$ prediction shown in Fig. 6b. This is again consistent with the mass extracted from the pion correlator at $\mu=0$ giving $a m_{\pi} / 2=0.1428(26)$ here for comparison. The sudden increase of the diquark condensate as compared to the $\chi \mathrm{PT}$ fit at around $1.6 \mu_{c}$ was also observed in [9] and interpreted as evidence of the BEC-BCS crossover. While this also agrees with the estimates from model predictions for the crossover region around $\mu \sim 0.8 m_{\pi}[7,8]$, we note however, that it might already be difficult to disentangle from the discretization artifacts setting in at larger $\mu$ as we will discuss below. The sharp drop of $\langle q q\rangle$ at $\mu$ around $7 \mu_{c}$ is close to half filling, where the quark-number density in lattice units reaches half its saturation value of maximum fermion occupancy on the finite lattice. Both condensates vanish at lattice saturation and other observables approach their quenched values.

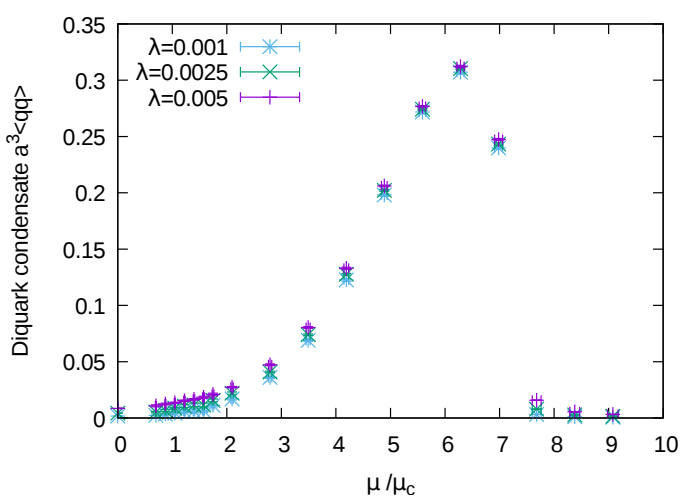

(a) $\mu$-dependence of $\langle q q\rangle$ for various $\lambda$.

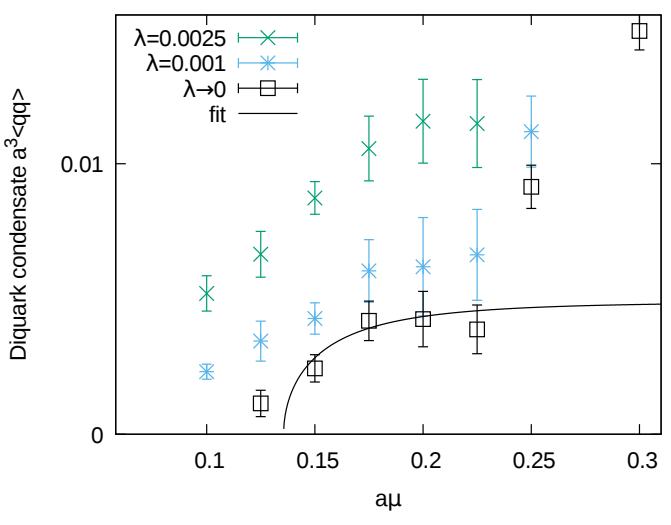

(b) $\lambda \rightarrow 0$ extrapolation of $\langle q q\rangle$ with $\chi \mathrm{PT}$ fit.

Figure 6: $\langle q q\rangle$ and leading-order $\chi \mathrm{PT}$ fit $\propto \sqrt{1-\left(\mu_{c} / \mu\right)^{4}}\left(16^{3} \times 32\right.$ lattice, am $\left.=0.01, \beta=1.7\right)$. 


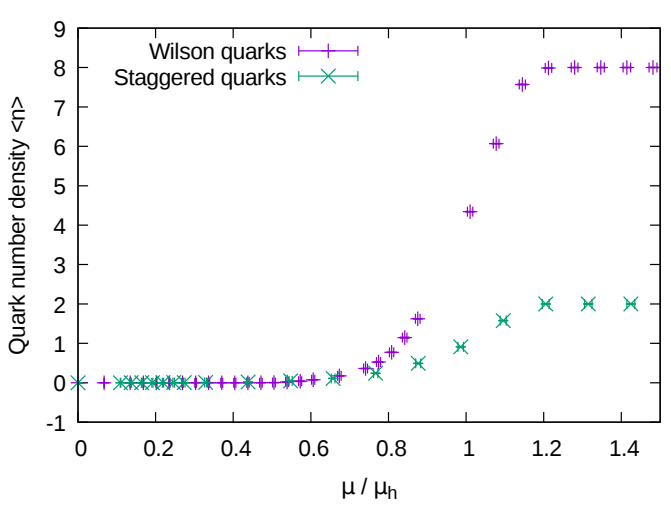

(a) quark-number density in lattice units.

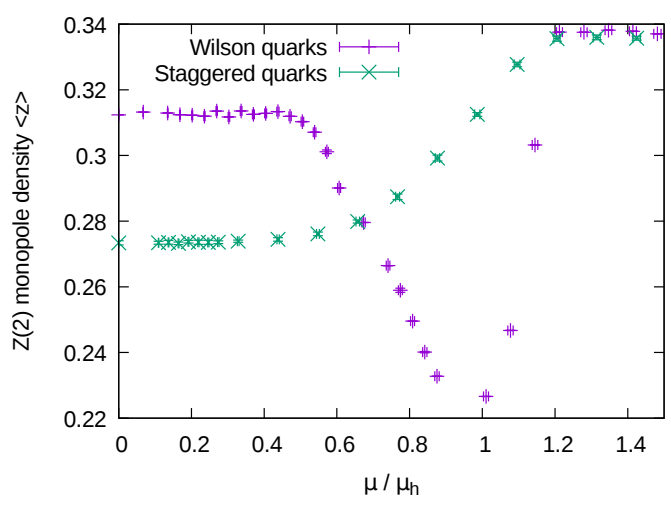

(b) $\mu$-dependence of $Z_{2}$-monopole density.

Figure 7: Comparison of two-flavor simulations from fourth-root staggered $\left(16^{3} \times 32\right.$ lattice, am = $0.01)$ and Wilson quarks $\left(10^{3} \times 20\right.$ lattice, $\left.\kappa=0.124689\right)$ at finite density with $\beta=1.7, a \lambda=0.001$.

\section{Staggered versus Wilson fermions at finite density}

The maximum number of fermions on a finite lattice depends on the fermion discretization, of course. In lattice units, the saturation density for Wilson fermions is $a^{3} n_{\text {sat }}^{\mathrm{W}}=2 N_{f} N_{c}=8$ in our two-flavor $\mathrm{QC}_{2} \mathrm{D}$ simulations, while for staggered fermions $a^{3} n_{\mathrm{sat}}^{\mathrm{KS}}=N_{c}=2$ here, independent of rooting. In order to compare the two discretizations without detailed mapping of the different scales we plot the respective quark-number densities over the chemical potential in units of its value $\mu_{\mathrm{h}}$ at half filling, where $n=n_{\text {sat }} / 2$ in Fig. 7a. When they start to deviate, this can be taken as a signal of strong discretization effects at least in one of the two.

As an example of observables which approache their quenched values with lattice saturation we plot the $Z_{2}$-monopole densities of both fermion discretizations in Fig. 7b. They start to show a notably different qualitative behavior at $\mu$ well below $\mu_{\mathrm{h}}$ already, before they approach saturation where their values are both consistent with the quenched result. The same is true for the Polyakov loop. It remains essentially independent of the chemical potential for staggered fermions as already observed in [9]. In contrast, it is well known from many previous studies of QCD-like theories with Wilson quarks [10, 18, 19], and the effective lattice theories for heavy quarks [11, 12], that the Polyakov loop starts to rise in the dense phase with a peak around half filling as also observed in Fig. 8 for our simulations with Wilson quarks on a $10^{3} \times 20$ lattice at $\beta=1.7, \kappa=0.124689$.

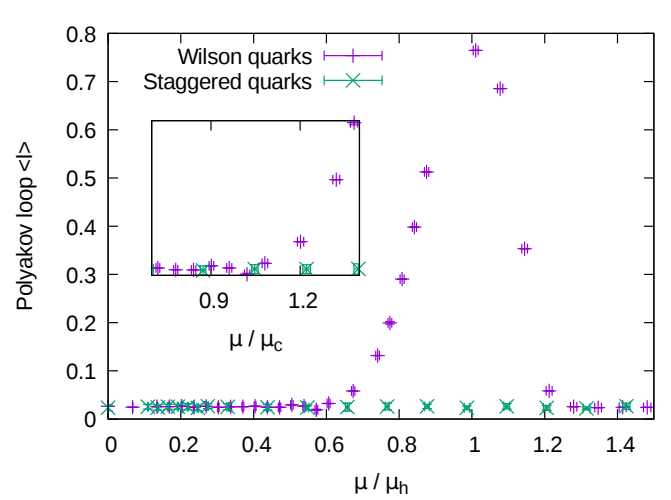

Figure 8: Polyakov loop with Wilson and staggered quarks, overview and inlay near $\mu_{c}$.

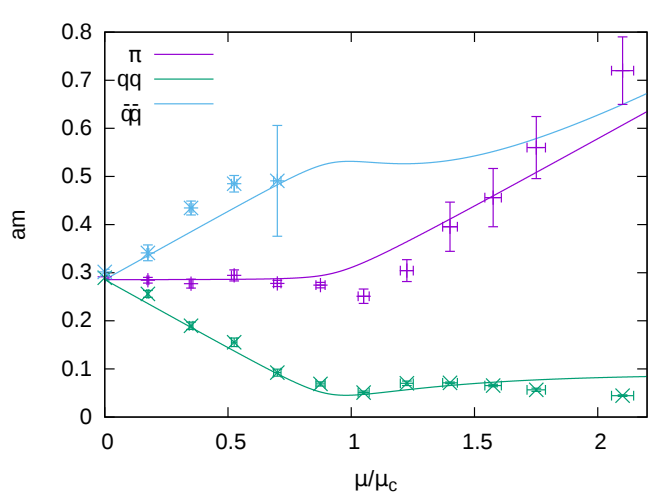

Figure 9: Goldstone spectrum outside bulk phase with continuum prediction from $\chi \mathrm{PT}$. 


\section{Summary and outlook}

We have simulated two-color QCD with fourth-root staggered quarks for two flavors at finite density. We have thereby compared results from lattice parameters employed previously at $\beta=$ 1.5 , deep inside the bulk phase with high $Z_{2}$-monopole density, with results from an improved gauge action together with a somewhat larger lattice coupling of $\beta=1.7$ where $Z_{2}$ monopoles are sufficiently suppressed to extract continuum physics. We found that the connected susceptibility subtraction used for the finite-temperature transition at low net-baryon density does not work for the $\mu$-dependent additive renormalization of the chiral condensate in the cold and dense matter. In the solid-state regime around the half-filled lattice we have observed significant differences between staggered and Wilson fermions, especially in the behavior of the Polyakov loop.

More importantly, however, our prelimiary Goldstone spectrum outside the bulk phase in Fig. 9 demonstrates that the continuum symmetry-breaking pattern of $\mathrm{QC}_{2} \mathrm{D}$ is recovered. Comparing the pion branches of Figs. 2 and 9 in the diquark-condensation phase we observe clear indications that the correct antiunitary symmetries of $\mathrm{QC}_{2} \mathrm{D}$ are restored also with staggered quarks in the continuum limit. The most likely reason is the realization of charge-conjugation invariance.

\section{References}

[1] S. Hands, J. B. Kogut, M.-P. Lombardo and S. E. Morrison, Nucl. Phys. B558 (1999) 327-346.

[2] J. B. Kogut, D. K. Sinclair, S. J. Hands and S. E. Morrison, Phys. Rev. D64 (2001) 094505.

[3] D. T. Son and M. A. Stephanov, Phys. Rev. Lett. 86 (2001) 592-595.

[4] J. B. Kogut, M. A. Stephanov, D. Toublan, J. J. M. Verbaarschot and A. Zhitnitsky, Nucl. Phys. B582 (2000) 477-513.

[5] J. B. Kogut, D. Toublan and D. K. Sinclair, Phys. Rev. D68 (2003) 054507.

[6] T. Kanazawa, T. Wettig and N. Yamamoto, JHEP 12 (2011) 007.

[7] N. Strodthoff, B.-J. Schaefer and L. von Smekal, Phys. Rev. D85 (2012) 074007.

[8] K. Kamikado, N. Strodthoff, L. von Smekal and J. Wambach, Phys. Lett. B718 (2013) 1044-1053.

[9] V. V. Braguta, E. M. Ilgenfritz, A. Yu. Kotov, A. V. Molochkov and A. A. Nikolaev, Phys. Rev. D94 (2016) 114510.

[10] T. Boz, P. Giudice, S. Hands, J.-I. Skullerud and A. G. Williams, AIP Conf. Proc. 1701 (2016) 060019.

[11] P. Scior and L. von Smekal, Phys. Rev. D92 (2015) 094504.

[12] O. Philipsen, for Confinement XII, Thessaloniki, Greece 2016, arXiv: 1612.03400.

[13] M. A. Clark, The Rational Hybrid Monte Carlo Algorithm, PhD thesis, Univ. of Edinburgh, 2005.

[14] I. G. Halliday and A. Schwimmer, Phys. Lett. B102 (1981) 337-340.

[15] P. Weisz and R. Wohlert, Nucl. Phys. B236 (1984) 397.

[16] D. Scheffler, Two-Color Lattice QCD with Staggered Quarks, PhD thesis, TU Darmstadt, 2015.

[17] W. Unger, The Chiral Phase Transition of QCD with $2+1$ Flavors, PhD thesis, Univ. Bielefeld, 2010.

[18] A. Maas, L. von Smekal, B. Wellegehausen and A. Wipf, Phys. Rev. D86 (2012) 111901.

[19] B. H. Wellegehausen, A. Maas, A. Wipf and L. von Smekal, Phys. Rev. D89 (2014) 056007. 